Editorial

\title{
SPECIAL TOPIC: SELECTED FINE PAPERS OF THE 2nd CONFERENCE ON MULTI-SOURCE IMPERFECT INFORMATION FUSION AND ITS APPLICATION
}

DOI $\quad 10.1007 / \mathrm{s} 11767-009-1003-5$

This Special Issue of the Journal of Electronics (China) is a selection of papers presented to the 2nd Multi-Source Imperfect Information Fusion (MIIF) and its Application Conference which was held from August 1, 2009. The Conference, bringing together researchers and practitioners from academia, industry, and government authorities, proved to be so popular that it has become an annual event to address the same objectives as the first one, by exchanging their research ideas and results and by discussing the state-of-the-art of methodology and algorithms of MIIF. The purposes of the special issue have not changed throughout the year, it focuses on all developments in the field of multi-source information, and reports on the exchange of knowledge from new observational, theoretical, experimental, and computational results, thus promoting the dissemination of information on all aspects of research and development in the field of information fusion. In this volume of Journal of Electronics (China), a collection of eight papers dealing with the theoretical analysis of MIIF as well as with the experiments and applications are issued, which highlight a number of features.

The multi-source information can be defined and modelled by one of the fuzzy set theory, rough set theory, Bayes reasoning, Dempster-Shafer evidence theory, possibility theory and conditional event algebra, etc. In recent years, researchers have explored the unification of the theories enabling the fusion of multi-source imperfect information and have finally considered random set theory. In the first paper, entitled "Random Sets: A Unified Framework for Multisource Information Fusion," by Xu Xiaobin et al., describes the close relationship between random set theory and other theories and presents several results to solve the challenge problems of how different types of imperfect in- formation can be unified in this framework. Furthermore, the paper gives some of the promising future trends and discusses briefly areas that need further investigations.

Updating or conditioning a body of evidence modelled within the l-Shafer's Theory (DST) plays an important role in most of Artificial Intelligence (AI) applications. Now, most of evidence updating rules are dependent on probability or Jeffrey's rule, but not fit for artificial intelligence inference. The next paper, "A New Evidence Updating Rule Based on Conditional Event," Wen Chenglin et al. presents a new evidence updating method based on the conditional event algebra.

The development of Dezert-Smarandache Theory (DSmT) arises from the necessity to overcome the inherent limitations of DST, and hence the third paper address issues related to the resolution of the bottleneck situation. "An Approximate Reasoning Method in Dezert-Smarandache Theory", by Li Xinde et al., suggests several methods in fast approximate reasoning where a set of assignments of the singletons components in hyper-power set can be grouped by bintree or tritree techniques.

The Probability Hypothesis Density (PHD) filter, an automatically track-managed multi-target tracker, is an ever attracting approach to tracking an unknown and time-varying number of targets in the presence of data association uncertainty, noise, clutter, and detection uncertainty in environments. The next three papers deal with the instability of algorithm's performance for target identity. "The Probability Hypothesis Density Filer with Evidence Fusion," by Liu Weifeng et al., analyzes a novel PHD filter with fuzzy logic-based evidence fusion and presents several future challenges to identifying a target. As mentioned above, traditional tracking algorithms are designed for assuming that the sensor provides a set of point 
measurements at each scan, therefore the applications of the algorithms are gained at the risk of losing part or all of the information desired. The next paper discusses the Signature Driven Multiple Target Tracking (SDT) method. "Signature Driven Multiple Target Tracking," by Sun Shuyan et al., describes one such method, utilizing the target signature in spectral, spatial and temporary spaces as well as the Markov property of target motion. The Multiple Hypothesis Tracking algorithm (MHT) and Interacting Multiple Model (IMM) filtering are generally accepted as the preferred for solving the problem of tracking multiple manoeuvring targets problem in cluttered environments. In the paper by Li Shuangquan et al., entitled "IMM/ MHT Fusing Feature Information in Visual Tracking," discusses a new radar implementation combining IMM with MHT algorithms that has been developed and tested by simulation. Tracking performance results are presented for the new algorithm, and preliminary estimates of computational requirements are given in order to demonstrate implementation feasibility.

The next paper, entitled "Classifier Fusion Based on Evidence Theory and its Application in Face Recognition," by Yang Yi et al., proposed a new method that implements multiple classifiers sion based on evidence combination and this utility has enabled a great increase in accuracy of face recognition.

The Dempster-Shafer's theory is an effective tool for dealing with uncertain information. It relies on the expert knowledge to provide evidence, requiring the evidence to be independent, and the applications of this theory are ultimately making their applications difficult and possibly ineffective. To solve this problem, the final paper, by Ye Qing et al., "An Intrusion Detection System Based on Evidence Theory and Rough Set Theory," outlines the rough sets theory to acquire independent evidence, and an illustrative example for hybrid intrusion detection system has shown that the proposed algorithm is feasible and effective in achieving performance improvement on the intrusion detection system.

We wish to thank the authors for having submitted and revised their manuscripts, as they have been a continuing source of satisfaction for us that the papers issued here would be consistently meeting the expectations of readers as good as ever. We also wish to acknowledge the assistance provided by the contributors to review that, without their expertise and advice, this Special Issue would have not attained the fine contents of high calibre. 